\title{
Improved excitation fidelity in cardiac imaging with 2-spoke parallel excitation at 7 Tesla
}

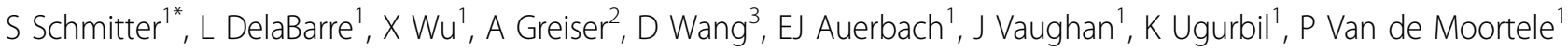 \\ From 16th Annual SCMR Scientific Sessions \\ San Francisco, CA, USA. 31 January - 3 February 2013
}

\begin{abstract}
Background
Cardiac MRI may greatly benefit from ultra high field (UHF) providing higher SNR and intrinsic contrast. But shorter RF wavelength yields transmit B1 (B1+) heterogeneity and contrast variations through the heart. These can be addressed by parallel transmission (pTX) using multispoke RF pulses as previously shown in other organs. However, applying pTX in cardiac MRI at 7T is challenging and requires rapid, multi-channel ECG triggered B1+ calibration and ECG synchronized, motion-insensitive pTX acquisitions. In this initial work we investigate the impact of 2-spoke RF pulse design on cardiac imaging at 7T using a 16-channel pTX system
\end{abstract}

\section{Methods}

Healthy volunteers were imaged at 7T (Siemens, Erlangen) using a 16 channel pTX system with 16 channel transceiver body coil. All sequences were modified to enable both ECG triggering and full pTX capability. B1+ maps of each transmit (TX) channel were obtained using a fast estimation technique [1]. Each map was acquired within $484 \mathrm{~ms}$ during diastole. 1- and 2-spoke pTX RF pulses (SINC shape, BWTP 4) were designed based on magnitude least squares (MLS) optimization [2] aiming at homogeneous excitation in manually drawn ROI. 3 excitations were compared: a) 1-spoke (= standard excitation) with nonoptimized initial RF phase setting (equal amplitude), b) 1-spoke with optimized RF magnitude/phase and c) 2-spoke RF pulse with magnitude/phase optimized for each spoke and channel. To keep a constant RF duration 1-spoke pulses were set to 1600us duration while 2-spoke subpulses were set to 800us. Coefficient of variation (CV, i.e. std/mean) in target ROI, total RF energy and peak energy per channel were computed for all RF pulses for same flip angle. Axial- and 4-chamber views were acquiried using a CINE GRE sequence (resolution 1.2x1.2x5mm3; 1 spoke: TR/TE $=41.7 / 2.5 \mathrm{~ms}, 32$ phases; 2 spoke: $\mathrm{TR} / \mathrm{TE}=44.8 / 2.7 \mathrm{~ms}, 29$ phases)

\section{Results}

Fig.1a shows axial views of predicted B1+ maps and GRE cardiac images using 1- and 2-spokes RF pulses. Signal patterns are highly consistent with predictions based on measured $\mathrm{B} 1+$ maps, including in more posterior regions (e.g. see aorta; also Fig.1b). Homogeneity improved with 2-spoke, with CV dropping from $16.4 \%$ to $10.0 \%$ with only minor increase in RF energy $(+12 \%$ - see Figure 2.1$)$. Fidelity improvement with 2- vs. 1-spoke pulses occured especially close to the ROI edges and posteriorly. Fig. 1b shows GRE images obtained with 2-spoke excitation in 4-chamber view at different cycle phases. $1+2$ spokes acquisition in 4 chamber view resulted in higher residual inhomogeneity and higher RF compared to axial views (Figure 2), likely due to larger ROI size and/or orientation. Note the absence of significant excitation pattern change through the cycle.

\section{Conclusions}

pTX 2-spoke excitation in the heart at 7T is feasible, providing improved excitation fidelity, and reduced peak RF amplitude and reduced total RF energy.

\section{Funding}

P41 EB015894, S10RR026783, R21-EB009138, KECK

Foundation. 


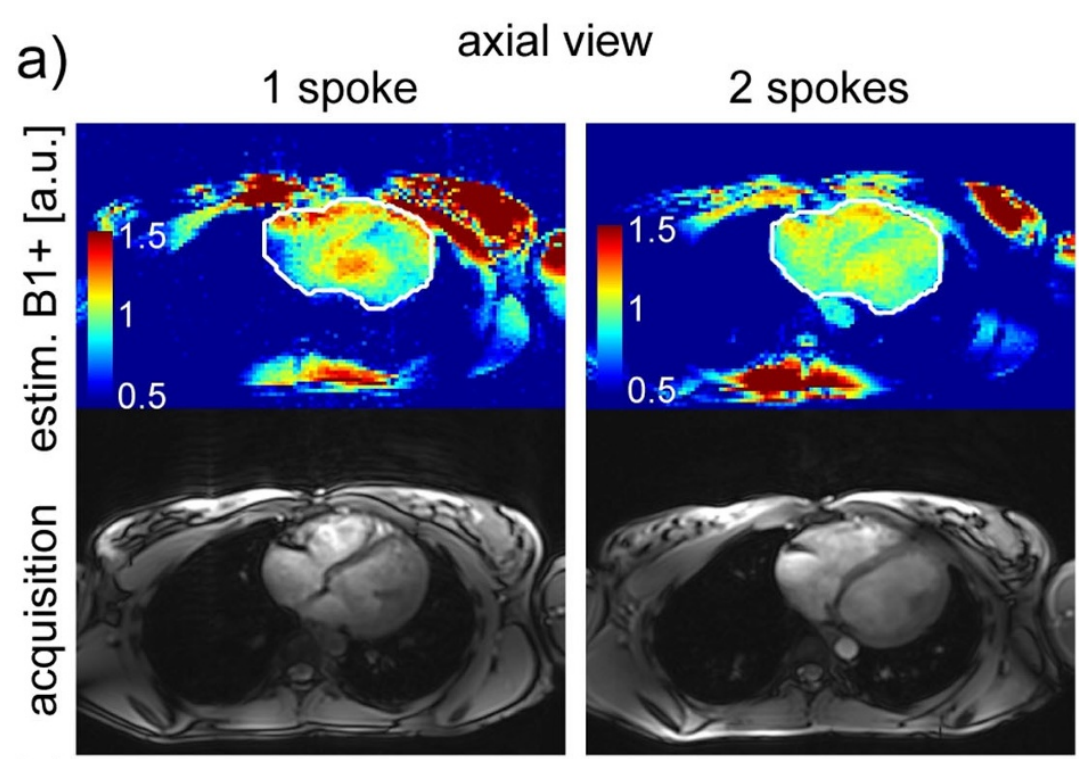

b)

2 spokes, 4 chamber view

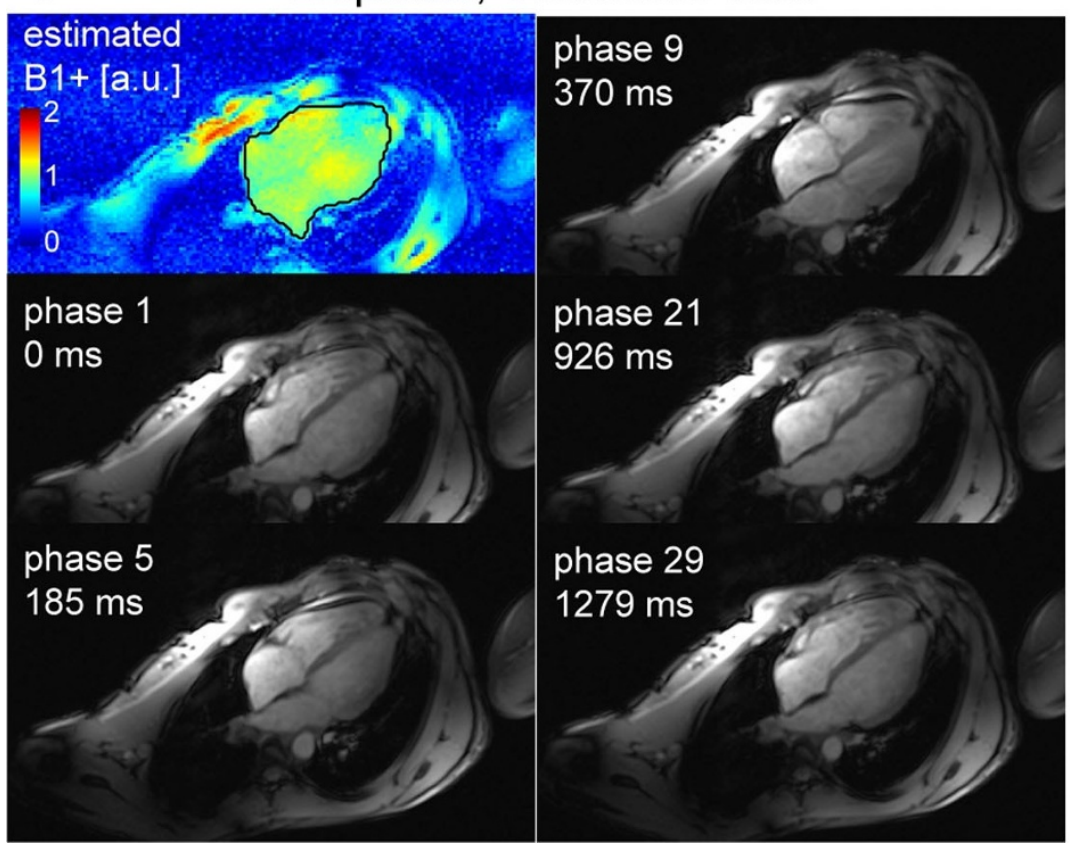

Figure 1 a) estimated B1+ maps for 1 and 2 spoke excitation (upper row) and last cardiac phase of corresponding CINE acquisition (lower row); b) 4 chamber view of different cardiac phases, acquired in CINE mode with 2 spokes at 7T. Upper left corner: corresponding B1+ estimation map (scale different to a) to appreciate overall pattern).

\begin{tabular}{|c||c|c|c|c||c|c|}
\hline \multicolumn{1}{|c||}{} & \multicolumn{5}{c||}{ axial view } & 4 chamber view \\
\cline { 2 - 7 } & $\begin{array}{c}\text { Non- } \\
\text { optimized }\end{array}$ & 1 spoke & 2 spoke & 2 spoke & 1 spoke & 2 spoke \\
\hline CV & $47.4 \%$ & $16.4 \%$ & $10.0 \%$ & $10.4 \%$ & $15.2 \%$ & $12.3 \%$ \\
\hline Energy & $228 \%$ & $100 \%$ & $112 \%$ & $100 \%$ & $170 \%$ & $170 \%$ \\
\hline $\begin{array}{l}\text { max(Energy } \\
\text { per Channel) }\end{array}$ & $48 \%$ & $100 \%$ & $60 \%$ & $55 \%$ & $129 \%$ & $102 \%$ \\
\hline
\end{tabular}

Figure 2 Summarized results of excitation fidelity, RF energy and max. of energy per channel. Gray shaded columns correspond to Fig.1. 


\section{Author details}

${ }^{1}$ Center for Magnetic Resonance Res earch, University of Minnesota, Minneapolis, MN, USA. ${ }^{2}$ Siemens Healthcare Sector, Erlangen, Bavaria, Germany. ${ }^{3}$ Siemens Medical Solutions USA, Inc.; Center for Magnetic Resonance Research, University of Minnesota, Minneapolis, MN, USA.

Published: 30 January 2013

\section{References}

1. Van de Moortele: ISMRM 2009.

2. Setsompop, et al: MRM 2008.

doi:10.1186/1532-429X-15-S1-W1

Cite this article as: Schmitter et al: Improved excitation fidelity in cardiac imaging with 2-spoke parallel excitation at 7 Tesla. Journal of Cardiovascular Magnetic Resonance 2013 15(Suppl 1):W1.

Submit your next manuscript to BioMed Central and take full advantage of:

- Convenient online submission

- Thorough peer review

- No space constraints or color figure charges

- Immediate publication on acceptance

- Inclusion in PubMed, CAS, Scopus and Google Scholar

- Research which is freely available for redistribution

Submit your manuscript at www.biomedcentral.com/submit

() Biomed Central 